\title{
Recovery from optic neuritis is associated with a change in the distribution of cerebral response to visual stimulation: a functional magnetic resonance imaging study
}

David J Werring, Edward T Bullmore, Ahmed T Toosy, David H Miller, Gareth J Barker, David G MacManus, Michael J Brammer, Vincent P Giampietro, Adriana Brusa, Peter A Brex, Ivan F Moseley, Gordon T Plant, W Ian McDonald, Alan J Thompson

NMR Research Unit, Institute of Neurology, Queen Square, London WC1N 3BG, UK

D J Werring

A T Toosy

D H Miller

G J Barker

D G MacManus

A Brusa

P A Brex

I F Moseley

G T Plant

W I McDonald

A J Thompson

Brain Image Analysis Unit, Institute of Psychiatry, De

Crespigny Park, London SE5 8AF, UK

E T Bullmore

M J Brammer

V P Giampietro

Correspondence to: Professor AJ Thompson, Institute of Neurology, Queen Square, London WC1N 3BG, UK email A.Thompson@ ion.ucl.ac.uk

Received 19 May 1999 and in final form

23 November 1999

Accepted 3 December 1999

\begin{abstract}
Objectives-Recovery to normal or near normal visual acuity is usual after acute demyelinating optic neuritis, despite the frequent persistence of conduction abnormalities as evidenced by the visual evoked potential (VEP). This raises the possibility that cortical adaptation to a persistently abnormal input contributes to the recovery process. The objective of this study was to investigate the pattern of cerebral response to a simple visual stimulus in recovered patients in comparison to normal subjects.

Methods-Functional magnetic resonance imaging (fMRI) was used to study the brain activation pattern induced by a periodic monocular $8 \mathrm{~Hz}$ photic stimulus in seven patients who had recovered from a single episode of acute unilateral optic neuritis, and in seven normal controls. VEPs and structural optic nerve MRI were performed on patients.

Results-Stimulation of either eye in controls activated only the occipital visual cortex. However, in patients, stimulation of the recovered eye also induced extensive activation in other areas including the insula-claustrum, lateral temporal and posterior parietal cortices, and thalamus; stimulation of the clinically unaffected eye activated visual cortex and right insulaclaustrum only. The volume of extraoccipital activation in patients was strongly correlated with VEP latency $(r=0.71$, $\mathbf{p}=\mathbf{0 . 0 0 5}$ ).

Conclusions-The extraoccipital areas that were activated in patients all have extensive visual connections, and some have been proposed as sites of multimodal sensory integration. The results indicate a functional reorganisation of the cerebral response to simple visual stimuli after optic neuritis that may represent an adaptive response to a persistently abnormal input. Whether this is a necessary part of the recovery process remains to be determined.

(F Neurol Neurosurg Psychiatry 2000;68:441-449)
\end{abstract}

Keywords: functional magnetic resonance imaging; visual recovery; demyelinating optic neuritis
One of the remarkable features of demyelinating diseases, both central and peripheral, is their capacity for complete recovery of function despite the persistence of gross distortions of the normal pattern of nerve conduction velocities. ${ }^{1-4}$ This is seen in optic neuritis, which in the acute stage is associated with a variable degree of conduction block and marked delay in the visual evoked potential (VEP). Recovery of visual acuity is associated with reversal of conduction block and is maintained despite, usually, the persistence of delays in the VEP.

It is clear that the CNS has somehow adapted so that it can deal effectively with a temporally distorted pattern of input. It seems likely that these adaptive changes take place at least in part in the synaptic relays in the cerebral cortex. What is the mechanism? We have taken a first step towards answering this question by posing another, simpler, one, and using functional magnetic resonance imaging (fMRI) to answer it: "In patients who have recovered from optic neuritis, is there a change in the distribution of activity in the cerebrum in response to a simple visual stimulus, compared with normal subjects?" By stimulating the affected and unaffected eyes in each patient in separate experiments, the activation patterns from affected and unaffected eyes may also be compared.

Optic neuritis has particular value for investigating adaptive responses to focal demyelination as it represents a single anatomically well defined lesion that is readily evaluated using clinical and electrophysiological measures. We have carefully selected patients who have recovered from a single episode of optic neuritis but who do not have clinical or neuroradiological evidence of disease elsewhere in the CNS. Thus, the confounding effects of multiple disseminated lesions in the brain are avoided, allowing the fMRI results to be more easily interpreted. A short account of some of this work has already appeared. ${ }^{5}$

\section{Patients and methods}

SUBJECTS

We studied seven patients (mean age 37.8 years; three men, four women) who had attended The National Hospital for Neurology and Neurosurgery or The Moorfields Eye Hospital with a single episode of typical unilateral acute optic neuritis diagnosed according to 
Table 1 Characteristics of patients

\begin{tabular}{|c|c|c|c|c|c|c|c|c|c|c|c|c|c|c|}
\hline \multirow[b]{2}{*}{ Age } & \multirow[b]{2}{*}{ Sex } & \multirow{2}{*}{$\begin{array}{l}\text { Years from } \\
\text { diagnosis }\end{array}$} & \multirow{2}{*}{$\begin{array}{l}\text { Side of } \\
\text { affected eye }\end{array}$} & \multirow{2}{*}{$\begin{array}{l}\text { Worst } \\
\text { documented } \\
\text { visual acuity }\end{array}$} & \multirow{2}{*}{$\begin{array}{l}\text { Brain } \\
\text { MRI }\end{array}$} & \multicolumn{2}{|c|}{$\begin{array}{l}\text { Acuity on day } \\
\text { of study }\end{array}$} & \multicolumn{2}{|c|}{$\begin{array}{l}\text { Colour vision on } \\
\text { day of study }\end{array}$} & \multirow{2}{*}{$\begin{array}{l}\text { MRI optic } \\
\text { nerves }\end{array}$} & \multicolumn{2}{|c|}{$\begin{array}{l}\text { VEP latency at } \\
\text { presentation }(\mathrm{ms})\end{array}$} & \multicolumn{2}{|c|}{$\begin{array}{l}\text { VEP latency at follow } \\
\text { up (ms) }\end{array}$} \\
\hline & & & & & & $R$ & $L$ & $R$ & $L$ & & Affected & Unaffected & Affected & Unaffected \\
\hline 38 & $\mathrm{~F}$ & 14 & $\mathrm{R}$ & $6 / 9$ & Normal & $6 / 5$ & $6 / 5$ & $17 / 17$ & $17 / 17$ & Abnormal (R) & 119.0 & 108.0 & 101.1 & 97.1 \\
\hline 49 & M & 13 & $\mathrm{~L}$ & $6 / 36$ & Normal & $6 / 5$ & $6 / 5$ & $17 / 17$ & $16 / 17$ & Normal & 114.0 & 112.5 & 103.1 & 101.6 \\
\hline 31 & $M$ & 0.5 & $\mathrm{~L}$ & $6 / 18$ & Normal & $6 / 5-2$ & $6 / 5-2$ & $17 / 17$ & $15 / 17$ & Abnormal (L) & ND & ND & 121.5 & 102.6 \\
\hline 33 & $\mathrm{~F}$ & 12 & $\mathrm{~L}$ & $6 / 60$ & Normal & $6 / 4$ & $6 / 4$ & $16 / 17$ & $15 / 17$ & Abnormal (L) & 147.0 & 106.0 & 99.6 & 98.6 \\
\hline 35 & M & 3 & $\mathrm{~L}$ & $6 / 24$ & Normal & $6 / 5-2$ & $6 / 6$ & $16 / 17$ & $15 / 17$ & Abnormal (L) & ND & ND & 118.6 & 103.3 \\
\hline 34 & $M$ & 1 & $\mathrm{~L}$ & NPL & Normal & $6 / 4$ & $6 / 5$ & $17 / 17$ & $16 / 17$ & Normal & ND & ND & 97.2 & 96.2 \\
\hline 45 & $\mathrm{~F}$ & 12 & $\mathrm{~L}$ & $\mathrm{CF}$ & Normal & $6 / 5$ & $6 / 5$ & $17 / 17$ & $15 / 17$ & Abnormal (L) & No pattern & 107.0 & 98.1 & 96.6 \\
\hline
\end{tabular}

$\mathrm{NPL}=$ No perception of light; $\mathrm{CF}=$ counting fingers; $\mathrm{ND}=$ not done; acuity=smallest type seen on the Snellen chart; colour vision=number of Ishihara plates correctly identified.

Table 2 Main areas of activation in generic brain activation maps for patients and controls

\begin{tabular}{|c|c|c|c|c|c|c|}
\hline \multirow[b]{2}{*}{ Area of activation } & \multirow{2}{*}{$\begin{array}{l}\text { Approximate } \\
\text { Brodmann } \\
\text { area }(B A)\end{array}$} & \multicolumn{3}{|c|}{ Talairach coordinates $(\mathrm{mm})$} & \multirow[b]{2}{*}{$F P Q$} & \multirow{2}{*}{$\begin{array}{l}\text { Cluster size } \\
\text { (voxels) }\end{array}$} \\
\hline & & $x$ & $y$ & $z$ & & \\
\hline \multicolumn{7}{|c|}{ Control subjects: left eye stmulation: } \\
\hline Visual cortex & $17,18,19$ & +11 & -65 & +4 & 10.4 & 253 \\
\hline Visual cortex & 17 & +9 & -63 & +9 & 13.8 & 177 \\
\hline Visual cortex & 18,19 & -13 & -56 & -2 & 6.2 & 99 \\
\hline Visual cortex & 17,18 & +11 & -82 & -2 & 8.1 & 143 \\
\hline Visual cortex & 17,18 & +6 & -68 & +15 & 7.6 & 104 \\
\hline Visual cortex & 19 & -9 & -70 & -2 & 5.7 & 67 \\
\hline \multicolumn{7}{|c|}{ Control subjects: right eye stimulation: } \\
\hline Visual cortex & $17,18,19$ & -11 & -72 & -2 & 10.6 & 257 \\
\hline Visual cortex & 17,18 & -9 & -72 & +4 & 13.9 & 230 \\
\hline Visual cortex & 18,19 & -2 & -74 & +9 & 7.8 & 175 \\
\hline Visual cortex & 17,18 & 0 & -75 & +15 & 6.7 & 53 \\
\hline Visual cortex & 17,18 & -17 & -65 & -7 & 5.8 & 49 \\
\hline Visual cortex & 19 & +17 & -58 & -7 & 7.2 & 33 \\
\hline \multicolumn{7}{|c|}{$\begin{array}{l}\text { Optic neuritis: unaffected eye } \\
\text { stimulation: }\end{array}$} \\
\hline Visual cortex & 17,18 & 0 & -74 & +4 & 10.5 & 195 \\
\hline Visual cortex & 17,18 & -17 & -67 & -2 & 5.6 & 160 \\
\hline Visual cortex & 17,18 & -11 & -60 & +15 & 7.0 & 121 \\
\hline Visual cortex & 18,19 & -15 & -58 & -7 & 5.6 & 94 \\
\hline Visual cortex & 17 & 0 & -74 & +9 & 6.4 & 92 \\
\hline Visual cortex & 17,18 & 0 & -75 & -2 & 8.6 & 91 \\
\hline Visual cortex & 18 & -15 & -60 & +9 & 5.8 & 56 \\
\hline Visal cortex & 19 & -19 & -51 & -13 & 4.5 & 37 \\
\hline Visual cortex & 19 & +17 & -53 & -13 & 4.1 & 25 \\
\hline Visual cortex & 18 & -2 & -74 & +20 & 5.0 & 23 \\
\hline Insula & $14-16$ & +36 & +28 & +4 & 3.5 & 21 \\
\hline \multicolumn{7}{|c|}{ Optic neuritis: affected eye stimulation: } \\
\hline Visual cortex & 17,18 & +8 & -58 & +4 & 7.0 & 371 \\
\hline Visual cortex & 17,18 & +13 & -56 & +9 & 7.8 & 329 \\
\hline Visual cortex & 18 & 0 & -72 & +15 & 5.6 & 219 \\
\hline Visual cortex & 18 & +4 & -77 & -2 & 5.3 & 193 \\
\hline Visual cortex & 18 & +11 & -61 & -7 & 4.0 & 129 \\
\hline Visual cortex & 18,19 & -13 & -60 & -7 & 4.3 & 106 \\
\hline Visual cortex & 18 & +6 & -74 & +9 & 5.5 & 95 \\
\hline Visual cortex & 18 & -15 & -79 & -2 & 4.3 & 68 \\
\hline Insula-claustrum & $14-16$ & +32 & +2 & +15 & 3.5 & 78 \\
\hline Insula-claustrum & $14-16$ & +26 & +11 & +9 & 3.8 & 75 \\
\hline Insula-claustrum & $14-16$ & -36 & +28 & +9 & 3.8 & 71 \\
\hline Insula-claustrum & $14-16$ & -42 & +23 & +4 & 3.6 & 47 \\
\hline Insula-claustrum & $14-16$ & -38 & -4 & -2 & 3.2 & 46 \\
\hline Insula/corpus striatum & $14-16$ & +23 & +12 & -2 & 4.2 & 79 \\
\hline Corpus striatum & - & +11 & +18 & +15 & 4.2 & 123 \\
\hline Corpus striatum & - & -32 & +21 & +15 & 4.3 & 80 \\
\hline Lateral temporal cortex & 21,22 & +49 & -23 & +9 & 3.7 & 81 \\
\hline Lateral temporal cortex & 21,22 & 55 & -47 & +9 & 3.7 & 50 \\
\hline Lateral temporal cortex & 21,22 & 53 & -35 & +4 & 3.7 & 44 \\
\hline Posterior parietal cortex & 40 & 45 & -19 & +20 & 3.5 & 66 \\
\hline Posterior parietal cortex & 40 & -47 & -23 & +20 & 4.1 & 63 \\
\hline Orbitofrontal cortex & 11 & 55 & +11 & +15 & 3.6 & 63 \\
\hline Thalamus & - & +8 & -11 & +9 & 4.5 & 43 \\
\hline
\end{tabular}

FPQ is the fundamental power quotient (see text), a measure of the power of response for each cluster.

standard criteria. ${ }^{6}$ Seven normal volunteers (mean age 31.0 years; three men, four women) were also studied. All subjects gave informed written consent. The study was approved by the National Hospital ethics committee. Patients were evaluated by a consultant neuroophthalmologist at presentation. On the day of study, visual acuity was measured using the Snellen chart and colour vision was assessed using Ishihara colour plates. Whole field visual evoked potentials were recorded using pattern reversal as described elsewhere. ${ }^{7}$ Patients with abnormalities on brain MRI were excluded to avoid a contribution from such lesions to the fMRI response.

IMAGING

Structural MRI

Echoplanar gradient echo MR images were acquired using a 1.5 Tesla GE Signa Horizon Echospeed system (General Electric, Milwaukee, WI,USA) with a standard quadrature head coil. High resolution echoplanar T2 weighted near axial images of the whole brain were obtained $(\mathrm{TR}=6000 \mathrm{~ms}, \mathrm{TE}=40 \mathrm{~ms}$, matrix $256 \times 256$, FOV $24 \mathrm{~cm} \times 24 \mathrm{~cm}$ ), together with high resolution fat suppressed spin echo images of the optic nerves (FSE 3250/68, matrix $512 \times 512$, FOV $20 \mathrm{~cm}) .{ }^{8}$ The structural MRI brain and optic nerve scans were evaluated (with blinding to clinical details) for any abnormalities by a neuroradiologist (IFM).

\section{Functional MRI}

One hundred and twenty $\mathrm{T} 2{ }^{\star}$ weighted images depicting blood oxygenation level dependent (BOLD) contrast were acquired in each 8 minute experiment at each of 10 near axial non-contiguous $5 \mathrm{~mm}$ thick slices through visual cortex roughly parallel to the AC-PC line: TE, $40 \mathrm{~ms}$; TR, $4000 \mathrm{~ms}$; field of view, 24 $\mathrm{cm} \times 24 \mathrm{~cm}$; matrix size, $96 \times 96$; in plane resolution, $2.5 \mathrm{~mm}$; interslice gap, $0.5 \mathrm{~mm}$.

\section{EXPERIMENTAL DESIGN}

Subjects passively viewed a visual display which alternated periodically between 20 second epochs of two contrasting conditions: (A) red $8 \mathrm{~Hz}$ photic stimulation to the whole visual field was presented to one eye using lightproof goggles (Grass Instruments, Quincy, MA, USA Model SV10SB) whereas the other eye received no visual stimulation; (B) no visual stimulation (darkness) was presented to both eyes. In total, 12 cycles of alternation between the $\mathrm{A}$ and $\mathrm{B}$ conditions were presented over the course of each experiment; condition B (no stimulation) was always presented first. Each subject was studied once for each side of monocular stimulation; the order of experiments was randomly decided.

\section{ANALYSIS}

Images were corrected for head motion by a two stage procedure of realignment and regression described in detail elsewhere. ${ }^{9}$ There were no significant differences between 
Table 3 One way ANOVA comparison between generic brain activation maps for stimulation of the unaffected eye in patients after left optic neuritis, and for stimulation of the right eye in controls showing Talairach coordinates.

\begin{tabular}{|c|c|c|c|c|}
\hline & \multirow[b]{2}{*}{ Approximate $B A$} & \multicolumn{3}{|c|}{ Talairach coordinates } \\
\hline & & $x(\mathrm{~mm})$ & $y(\mathrm{~mm})$ & $z(\mathrm{~mm})$ \\
\hline \multicolumn{5}{|c|}{ Areas of significantly greater response in patients: } \\
\hline Right anterior insula & $14-16$ & -35 & +28 & +4 \\
\hline Right visual cortex & 19 & -27 & -54 & -1 \\
\hline Right visual cortex & 18 & -5 & -72 & -1 \\
\hline Left visual cortex & 18 & +20 & -63 & -1 \\
\hline Left visual cortex & 19 & +15 & -62 & -8 \\
\hline Left visual cortex & 18 & +10 & -62 & +4 \\
\hline Left visual cortex & 18 & +6 & -46 & +4 \\
\hline Left visual cortex & 18 & +16 & -70 & +4 \\
\hline Left visual cortex & 17 & +5 & -75 & +12 \\
\hline Left visual cortex & 18 & +5 & -81 & +12 \\
\hline Right lateral temporal cortex & 22 & -48 & -5 & +8 \\
\hline Left lateral temporal cortex & 22 & +49 & -22 & +8 \\
\hline Right cingulate gyrus & 31 & -7 & -52 & +8 \\
\hline Right cingulate gyrus & 31 & -8 & -63 & +8 \\
\hline Left cingulate gyrus & 31 & +7 & -65 & +12 \\
\hline Right cingulate gyrus & 23 & -25 & -53 & +8 \\
\hline Right inferior frontal gyrus & 45 & -38 & +24 & +4 \\
\hline \multicolumn{5}{|c|}{ Areas of significantly greater response in controls: } \\
\hline Right visual cortex & 19 & -18 & -62 & -8 \\
\hline Right visual cortex & 19 & -32 & -61 & -8 \\
\hline Left visual cortex & 18 & +11 & -75 & -8 \\
\hline Right visual cortex & 17 & -11 & -92 & -1 \\
\hline Right visual cortex & 18 & -10 & -82 & -1 \\
\hline Right visual cortex & 18 & -16 & -70 & -1 \\
\hline Left visual cortex & 17 & +5 & -94 & -1 \\
\hline Left visual cortex & 18 & +7 & -80 & -1 \\
\hline Left visual cortex & 19 & +12 & -60 & -1 \\
\hline Left visual cortex & 19 & +18 & -42 & -1 \\
\hline Right visual cortex & 18 & -34 & -78 & +4 \\
\hline Right visual cortex & 18 & -9 & -82 & +4 \\
\hline Left visual cortex & 17 & +12 & -95 & +4 \\
\hline Left visual cortex & 18 & +7 & -75 & +4 \\
\hline Right visual cortex & 17 & -10 & -82 & +8 \\
\hline Right visual cortex & 17 & -10 & -75 & +8 \\
\hline Left visual cortex & 17 & +10 & -75 & +8 \\
\hline Right visual cortex & 18 & -10 & -82 & +12 \\
\hline
\end{tabular}

Search volume 1476 voxels, $p<0.05$. Patients $>$ controls $=125$ voxels, controls $>$ patients $=305$. voxels.

patients and comparison subjects for stimulus correlated motion, which was estimated by the power of a periodic trend at the frequency of $\mathrm{AB}$ alternation in the time series of rotations and translations of the head in three dimensions. ${ }^{9}$ The standardised power of experimentally determined signal change at the frequency of alternation between stimulation and baseline conditions, or fundamental power quotient (FPQ), in each movement corrected fMRI time series was estimated by sinusoidal regression. ${ }^{10}$ The FPQ represents the sum of squared amplitudes of a sine and cosine wave at the frequency of $\mathrm{AB}$ alternation, divided by its standard error (see Bullmore et $a l^{10}$ for further details). These sine and cosine waves at $\mathrm{AB}$ frequency were parameterised by coefficients $\gamma$ and $\delta$ respectively; the phase of periodic response in each time series was estimated by $\phi=\tan -1 \delta / \gamma .{ }^{11}$ Individual brain activation maps were examined to ensure that no subject showed an extreme pattern of response that might unduly influence the result of generic brain activation mapping. Individual fMRI time series were inspected at occipital and extraoccipital sites of activation. Generic brain activation maps were computed in the standard space of Talairach and Tournoux ${ }^{12}$ by a permutation test with voxel-wise one tailed probability of false positive error $=p<0.0001$. At this size of test, the expected number of false positive voxels in each map is less than two. Generically activated voxels were colour coded according to their phase of response and overlaid on a grey scale EPI template image to form a generic brain activation map (GBAM) ${ }^{13}$

The power of periodic response was compared between groups and between eyes within group by analysis of variance (ANOVA). The following one way ANOVA model was fitted at each intracerebral voxel in standard space:

$\mathrm{FPQ}=\mu+\beta . \mathrm{F}+\varepsilon$

where $\mu$ is the overall mean power at the ith voxel, $\mathrm{F}$ is a factor coding group membership (patient or control) or stimulated eye (right or left), and $\varepsilon$ is an error. The coefficient $\beta$ was tested by permutation at those voxels that were generically activated in one or both of the groups of data being compared. ${ }^{14}{ }^{15}$ The use of this permutation procedure for MRI data analysis is described in detail elsewhere. ${ }^{9}{ }^{14}$ For these analyses, we set the two tailed probability of false positive error $p=0.05$. This relatively lenient threshold is justified by the restricted search volume for between group comparisons. Where we have tested for a significant difference in the power of response between eyes (that is, we have used repeated measures), the permutation procedure has been adjusted accordingly.

We also investigated the relation between extent of abnormal (extraoccipital) activation and neurophysiological evidence for persistently delayed optic nerve conduction in the patients. The volume of extraoccipital activation was estimated using a semiautomated contouring algorithm (DispImage, D Plummer, UCL, London UK) in each individual brain activation map. The relation between extraoccipital volume of brain activation and VEP latency was assessed by scatter plot and by a significance test of Spearman's correlation coefficient.

\section{Results}

The patient characteristics are shown in table 1. All patients had a typical clinical history of acute optic neuritis with documented loss of visual acuity at presentation, and by the time of our study had recovered normal visual acuity and colour vision. All had normal eye movements, with accurate fixation during formal visual field perimetry. Four patients had VEP recordings at presentation; three were abnormal (two were delayed and one absent). All patients had VEP recordings at the time of our study. The three previously abnormal VEPs now had normal latencies; two of the remaining three cases (with shorter clinical histories) had delayed responses; MRI of the affected optic nerve was abnormal in five of the seven cases (fig 1).

There were no significant differences between the control and patient groups in stimulus correlated motion during the fMRI studies. Selected slices from generic brain activation maps $^{13}$ for patients and control subjects are shown in fig 2; coordinates of the main foci of activation in the generic brain activation maps in standard space, ${ }^{12}$ cluster sizes and FPQ values are given in table 2 . In the control subject generic brain activation maps (fig $2 \mathrm{~A}$ and B), activation in response to the stimulation of 
either eye was demonstrated almost exclusively in visual cortical areas (approximate Brodmann areas (BA) 17, 18, and 19). A visual impression of asymmetry in activation patterns between the left and right eyes (left eye stimulation apparently inducing greater right visual cortex activation, right eye stimulation inducing greater left visual cortex activation) was confirmed using a repeated measures one way ANOVA thresholded at $\mathrm{p}<0.05$.

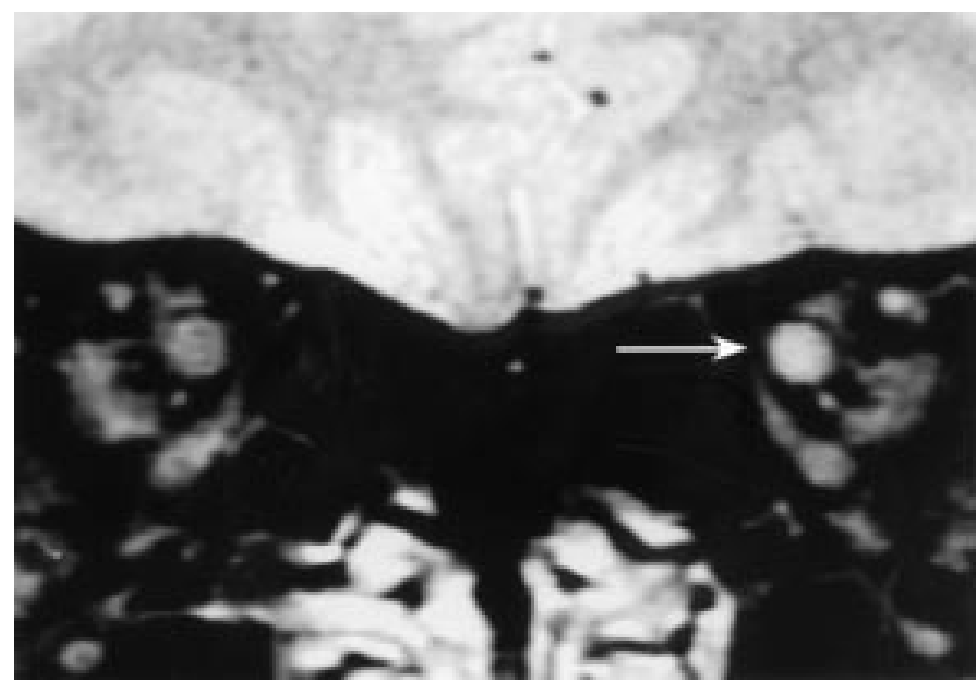

Figure 1 Coronal MRI through the optic nerves in a patient after a single episode of left unilateral optic neuritis 14 years previously. Note the abnormal high signal in the affected nerve (arrowed), indicating persisting structural abnormality despite the excellent clinical recovery found.

Table 4 One way ANOVA comparison between generic brain activation maps for affected eye stimulation in patients after left optic neuritis, and left eye stimulation in controls showing Talairach coordinates.

\begin{tabular}{|c|c|c|c|c|}
\hline & \multirow[b]{2}{*}{ Approximate $B A$} & \multicolumn{3}{|c|}{ Talairach coordinates } \\
\hline & & $x(m m)$ & $y(m m)$ & $z(\mathrm{~mm})$ \\
\hline \multicolumn{5}{|c|}{ Areas of significantly greater response in patients: } \\
\hline Right orbitofrontal cortex & 11 & -22 & +40 & -12 \\
\hline Right insula-claustrum & $14-16$ & -28 & +28 & -1 \\
\hline Left insula-claustrum & $14-16$ & +27 & +29 & -1 \\
\hline Right insula-claustrum & $14-16$ & -31 & +25 & +4 \\
\hline Left insula-claustrum & $14-16$ & +35 & +20 & +4 \\
\hline Right corpus striatum & - & -20 & +3 & -1 \\
\hline Right inferior frontal cortex & 45 & -52 & +26 & +4 \\
\hline Right visual cortex & 19 & -12 & -45 & -1 \\
\hline Right visual cortex & 18 & -14 & -55 & +4 \\
\hline Right visual cortex & 17 & -13 & -70 & +12 \\
\hline Right cingulate gyrus & 23 & -14 & -60 & +8 \\
\hline Right lateral temporal cortex & 22 & -49 & -25 & +8 \\
\hline Right cingulate gyrus & 23 & -13 & -61 & +12 \\
\hline Left cingulate gyrus & 23 & +8 & -61 & +12 \\
\hline \multicolumn{5}{|c|}{ Areas of significantly greater response in controls: } \\
\hline Left visual cortex & 18 & +21 & -64 & -8 \\
\hline Right visual cortex & 18 & -8 & -80 & -1 \\
\hline Right visual cortex & 19 & -12 & -55 & -1 \\
\hline Left visual cortex & 18 & +5 & -85 & -1 \\
\hline Left visual cortex & 18 & +11 & -77 & -1 \\
\hline Left visual cortex & 18 & +12 & -61 & -1 \\
\hline Left visual cortex & 19 & +25 & -61 & -1 \\
\hline Left visual cortex & 19 & +12 & -44 & -1 \\
\hline Right superior temporal gyrus & 22 & -53 & -20 & +4 \\
\hline Right middle temporal gyrus & 22 & -55 & -37 & +4 \\
\hline Right visual cortex & 18 & -32 & -78 & +4 \\
\hline Right visual cortex & 18 & -25 & -75 & +4 \\
\hline Left visual cortex & 17 & +5 & -85 & +4 \\
\hline Left visual cortex & 18 & +7 & -75 & +4 \\
\hline Left visual cortex & 18 & +10 & -61 & +4 \\
\hline Right visual cortex & 17 & -10 & -72 & +8 \\
\hline Left visual cortex & 17 & +6 & -75 & +8 \\
\hline Right visual cortex & 17 & -10 & -75 & +12 \\
\hline Left visual cortex & 18 & +20 & -80 & +16 \\
\hline
\end{tabular}

Search volume 2471 voxels; $p<0.05$. Patients $>$ controls $=125$ voxels; controls $>$ patients $=424$ voxels.
Stimulating the clinically unaffected eye in patients induced additional activation in the right insula-claustrum (BA 14) (fig 2 C). To confirm that this apparent visual difference between the group activation maps was of statistical significance, a one way ANOVA comparing the generic map for the patient's unaffected eyes (including only the six patients with a left optic neuritis to remove possible confounding effects of right-left fMRI activation asymmetry) with the generic map for the control right eye studies was performed (table 3). This confirmed increased activation in the right insula-claustrum region in patients compared with controls, but showed a significantly larger response in visual cortex (approximate BA $17,18,19)$ in controls.

In response to stimulating the recovered eye, additional extensive activations were seen bilaterally in the insula-claustrum (BA 14-16), lateral temporal cortex (BA 21 and 22), posterior parietal cortex (BA 39 and 40), orbitofrontal cortex (BA 11), corpus striatum, and thalamus (fig 2 D). A one way ANOVA comparison between the generic activation maps of the affected eye (again including only the six patients with a left optic neuritis) and the left eye maps from controls confirmed greater activation in patients in the orbitofrontal cortex, anterior insula bilaterally, and the corpus striatum (table 4), but significantly greater activation in controls in visual cortical areas (approximate BA 17,18,19). The finding of extraoccipital activation induced by stimulation of the affected eye in patients was seen in all seven individual activation maps, indicating that the group result was not influenced by a few outlier observations.

A generic brain activation map for stimulation of the affected eye in the two patients with definite VEP delay at the time of fMRI showed a much greater extent of extraoccipital activation than that for the remaining five patients without VEP delay (fig 3). Furthermore, whole field VEP latency showed a strong positive correlation with the volume of extraoccipital activation (Spearman's $r=0.71, \mathrm{p}=0.005$ ) (fig 4).

Whereas occipital regions were activated in the patient group with MR signal maximum during the photic stimulation condition, as in the control group, areas of extraoccipital activation showed a relatively delayed response with signal maximum during the early part of the dark condition; figures 2 and 5 .

\section{Discussion}

These findings show that there is indeed a change in the distribution of cortical activation by a simple visual stimulus in patients who have recovered from acute optic neuritis compared with normal subjects. Activation in response to periodic stimulation of the recovered eye occurs not only in the occipital visual cortex, but also in the insula-claustrum, lateral temporal, posterior parietal and orbito-frontal cortices, corpus striatum, and thalamus (fig $2 \mathrm{D}$, table 2). Although these sites are not normally activated by a simple visual stimulus, they are known to have extensive connections with visual processing areas, and some of them have 


\section{$-1.5$}

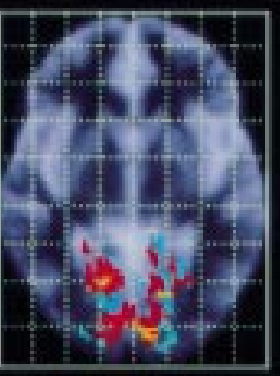

4
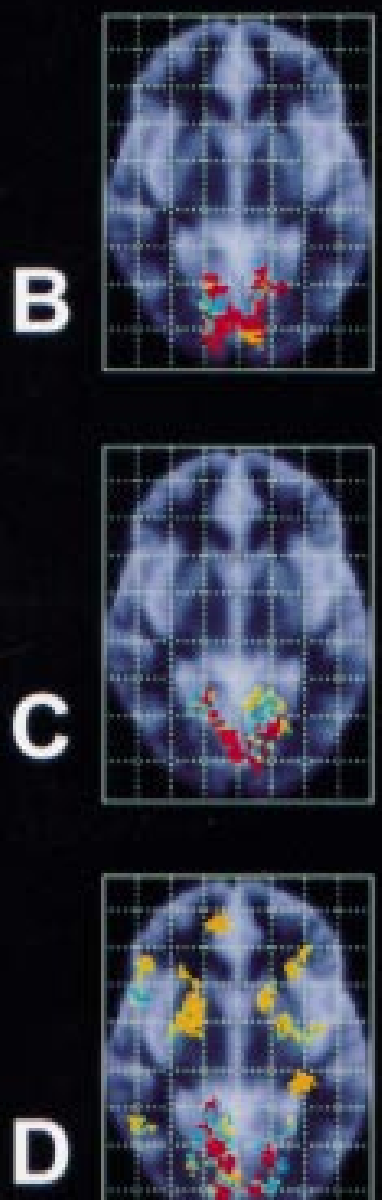

\section{$+4.0$}
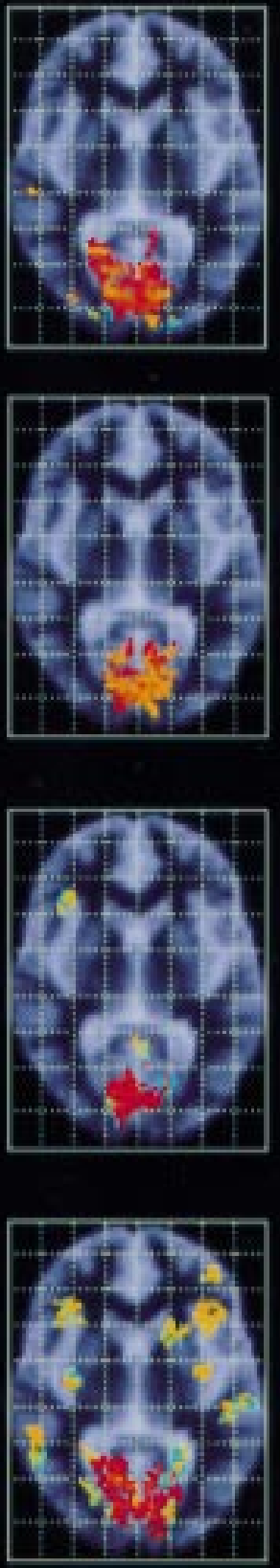

\section{$+9.5$}
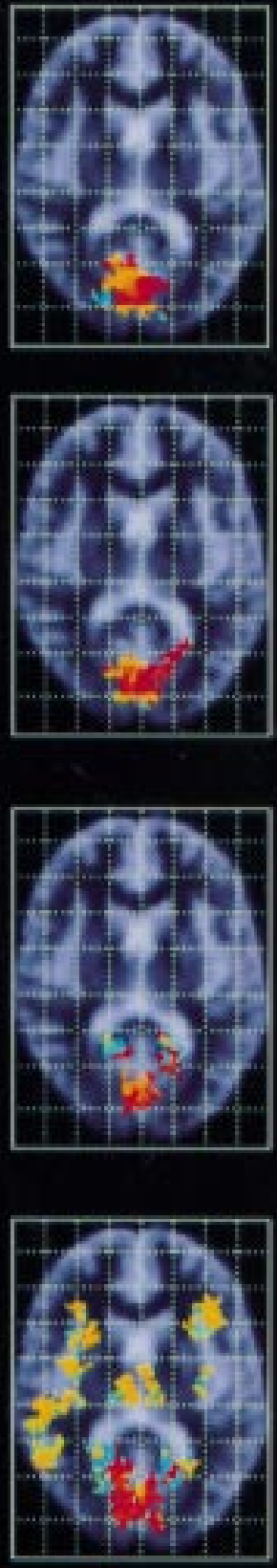

40

0

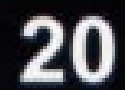

Figure 2 Generic brain activation maps from seven control subjects and seven patients showing areas of significant response to monocular photic stimulation compared with binocular darkness. The one tailed probability of false positive activation $p<0.0001$ for each voxel; activated voxels are colour coded according to the delay (in seconds) of periodic response relative to the onset of photic stimulation. The left side of each map represents the right side of the brain; $z$ coordinates in standard space are given for each slice in $\mathrm{mm}$. (A) In the control subject group (left eve) there is activation only in bilateral visual cortex, with a larger area of activation in the right compared with the left visual cortex. (B) The right eye response showed a similar pattern but with greater activation of the left visual cortex. (C) In the patient group (unaffected eye) there is an additional focus of activation in right insula-claustrum. (D) In the patient group (affected eye) there is additional activation of a network of multimodal processing areas including bilateral insula-claustrum, lateral temporal cortex, posterior parietal cortex, thalamus, and corpus striatum. Note that periodic response in extraoccipital areas is considerably delayed relative to the response in visual cortex. 


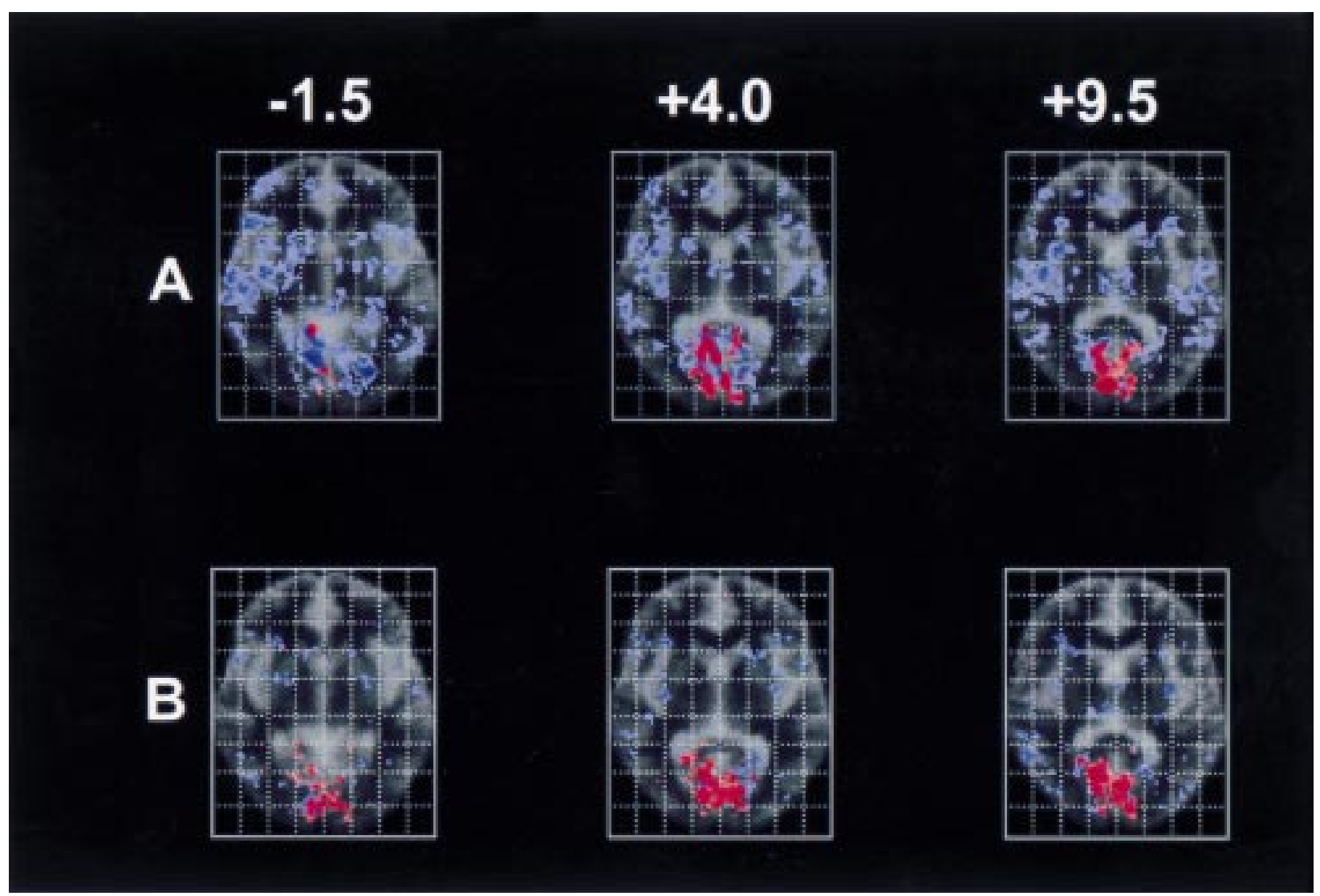

Figure 3 Generic brain activation maps from stimulation of the affected eye in patients after recovery from optic neuritis (A) with VEP delay (defined as a latency prolongation of $>109 \mathrm{~ms}$ for those under 40 years old and $>122 \mathrm{~ms}$ for those over 40 years old) and (B) without VEP delay. $z$ Coordinates for each slice in Talairach space are given in $\mathrm{mm}$. The phase of response is coded as red (maximum fMRI signal during the stimulus condition) or blue (maximum fMRI signal during the baseline condition). The deepness of colour represents the magnitude of the fundamental power quotient (FPQ) at each voxel, a measure of the power of periodic response at the experimental frequency. Note the similar distribution of extraoccipital areas activated in both maps, but the much greater extent in those with VEP delay.

been proposed as areas of multimodal sensory integration. ${ }^{16}$ The claustrum is a small nucleus, the function of which remains uncertain. It is well developed in primates, and its size increases roughly in proportion to the volume of cerebral cortex. ${ }^{17}$ It has complex projections to many cortical regions. It has rich connections with BA 17, 18, and 19 and the visual thalamus, ${ }^{17}$ and receives input from both visual fields ${ }^{18}$; furthermore the claustrum can functionally modulate the response properties of neurons in the primary visual cortex (BA 17). ${ }^{19}$ The claustrum receives input from, and projects to multimodal visual and auditory areas, and has recently been proposed as a site of transfer of information between tactile and visual modalities. ${ }^{20}$ These extensive visual connections provide a plausible physiological substrate for activation of the claustrum during simple visual stimulation. The insula has also been proposed as a multimodal convergence area, ${ }^{21}$ and damage to the insula in humans causes a syndrome of multimodal neglect. ${ }^{22}$ The anterior insula has substantial connections with the orbitofrontal cortex and the thalamus as well as limbic areas. ${ }^{21}$ The posterior insula has connections with frontal, temporal and parietal cortices, and the thalamus. ${ }^{21}$ The medial pulvinar thalamic nucleus in turn projects to higher order multimodal association cortex. $^{23}$ The lateral temporal cortex (BA 21 and 22) and posterior parietal cortex (BA 39

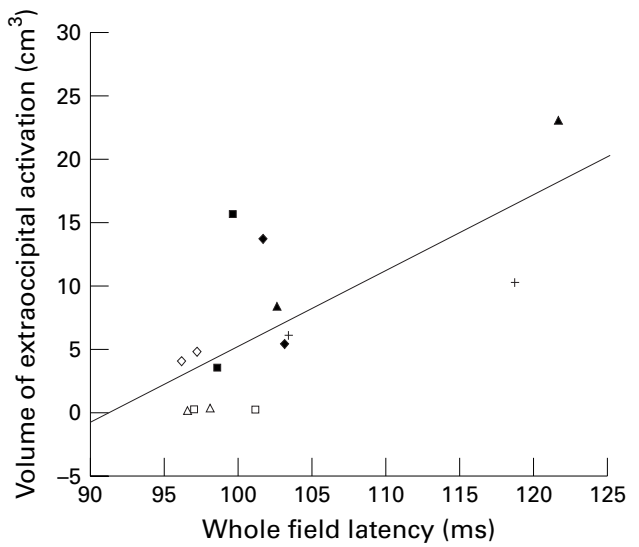

Figure 4 Correlation between the volume of extraoccipital activation and whole field VEP latency (including both affected and unaffected optic nerves) for seven patients after unilateral optic neuritis.

and 40) were activated in patients in response to simple visual stimulation of the affected eye, but not in normal controls. These areas have been suggested to be sites of multimodal sensory processing on the basis of homologies with primate multimodal areas. ${ }^{16}$ Finally there is experimental evidence that the corpus striatum, although classically thought to have mainly a motor role, receives functional input from the visual cortex. ${ }^{24}$

Thus the areas that are activated during stimulation of the affected eye in patients 

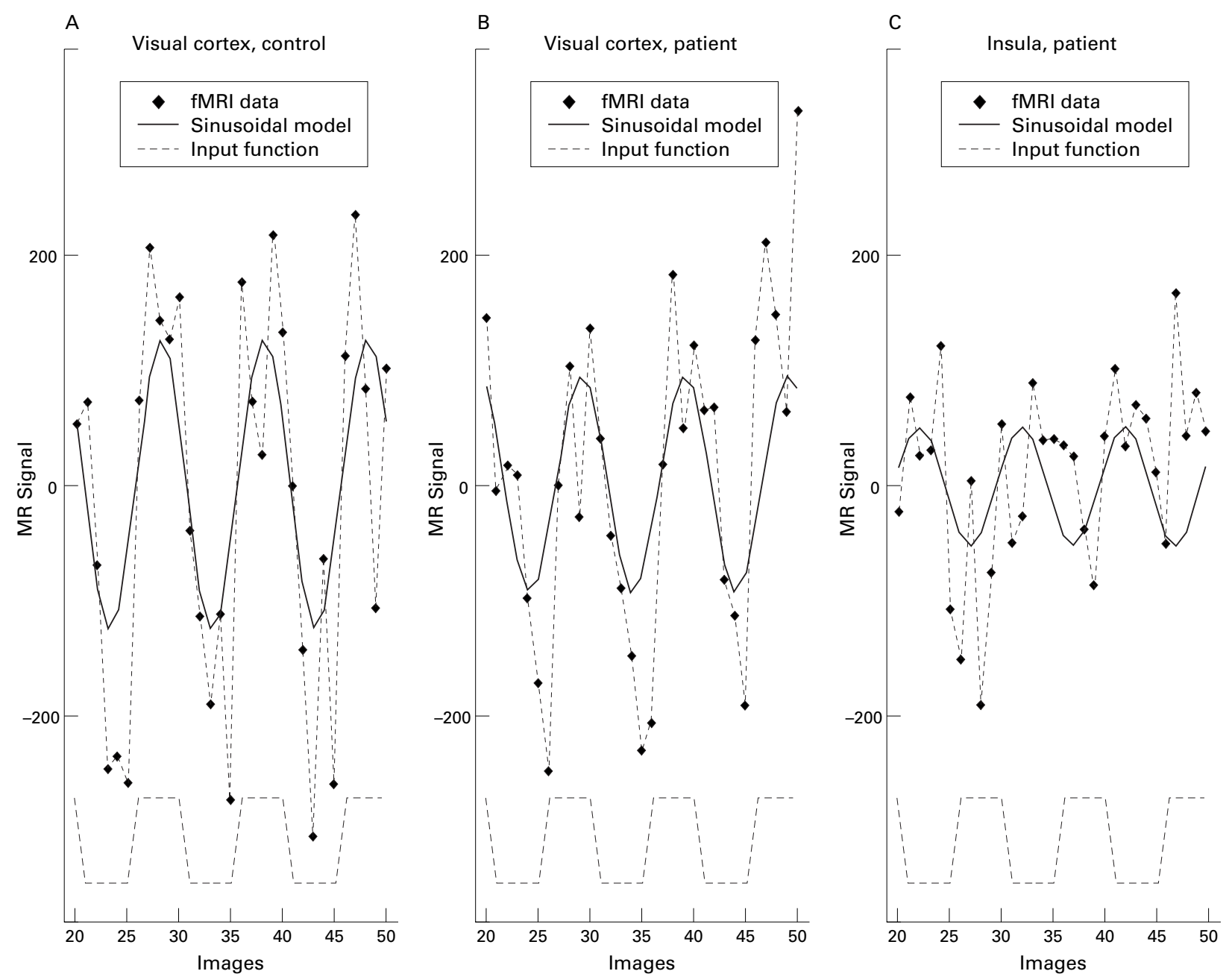

Figure 5 Regional mean fMRI time series from an individual brain activation map obtained from a 35 year old man who had fully recovered from an episode of left optic neuritis. In this experiment 20 second epochs of $8 \mathrm{~Hz}$ monocular visual stimulation to the recovered eye alternated with binocular darkness. The fMRI signal time series in the occipital visual cortex and in one of the areas activated outside the occipital cortex (left insula) are shown (diamonds). A periodic response to the stimulus (experimental input function; dotted square wave, bottom of figure) is seen in both regions, but is delayed in the left insula compared with the visual cortex.

constitute a cortical and subcortical network of areas known to be involved in visual or multimodal sensory processing. The activation map for the unaffected eye showed a limited activation of this network, confined to the right insula-claustrum region at the statistical threshold used in this study. This could be a response to abnormal input due to clinically silent abnormality of conduction in the unaffected optic nerve (a well recognised phenomenon). ${ }^{2}$ An alternative mechanism, suggested by the fact that visual input to the claustrum is binocular, ${ }^{18}$ is that a change in functional organisation in the claustrum due to disease in one eye could result in altered activation additionally on stimulating the other clinically unaffected eye. Why this activation is lateralised to the right side is uncertain, although it is noteworthy that six of seven unaffected eyes were on the right.

Although the significance of these areas of extraoccpital activation in the recovery process in optic neuritis remains to be determined, and the number of cases is small and must be increased, the data suggest that they may be related to the degree of VEP delay. Firstly, the greater extent of extraoccipital activation from stimulating the affected eye in patients with VEP delay compared with those without VEP delay suggests that slowed conduction in the optic nerve is a contributory factor in producing, and sustaining, an abnormal distribution of cerebral response to visual stimulation. Secondly, there is a strong correlation between the volume of extraoccipital activation and the VEP latency. The origin of a prolonged VEP latency is complex, but is likely to be in part due to slowed conduction in completely or partially demyelinated axons; a reduction and dispersion of the normal input due to unequal slowing in different fibres may also contribute. ${ }^{25}$ In the acute phase of optic neuritis there is a decrease in amplitude and an increase in the latency of the VEP. As recovery of vision occurs the amplitude recovers to a variable extent, but the prolonged latency persists for much longer, even when full visual recovery has usually occurred. ${ }^{25}$ Our findings suggest that the extraoccipital network activated in patients after optic neuritis may represent an adaptive cerebral response to VEP delay, which could contribute to the recovery process. 
The phase of periodic fMRI signal change in the extraoccipital areas activated only by patients was delayed relative to the phase of response to photic stimulation in visual cortex; figure $2 \mathrm{C}$ and $\mathrm{D}$ and fig 5 . On the assumption that locally increased BOLD magnetic resonance signal reflects increased synaptic activity, whether excitatory or inhibitory, ${ }^{11}$ there are several possible explanations for this finding. Firstly, extraoccipital multimodal areas could be excited by delayed propagation of neural activity from primary visual areas. The delay that we have found between occipital visual cortex and these areas (of about 10 seconds) is, however, considerably longer than delayed hierarchical excitation of visual cortical processing areas (shown by electrophysiological studies to be of the order of milliseconds), making this unlikely. A second possibility is that multimodal regions are actively inhibited in patients during the dark condition (causing locally increased fMRI signal), from which they are released (disinhibited) at the onset of photic stimulation. Disinhibition (by experimental reduction of inhibitory input to the superior colliculus, a putative subcortical multimodal region) has been suggested as a contributory mechanism in recovery from experimental injury to mammalian visual cortical areas (the Sprague effect), ${ }^{26}$ but the role of such a process in recovery from anterior visual pathway lesions has not been investigated. A third possibility is that extraoccipital areas show a relative increase in activity during the baseline condition that may be suspended during visual stimulation. Finally, the peak fMRI signal could occur later in multimodal areas due to a different, delayed haemodynamic reponse. There is evidence that the response to a semantic decision task in multimodal lateral frontal areas in normal subjects is delayed by about 10 seconds compared with the response to pure tone discrimination in primary auditory cortex. ${ }^{27}{ }^{28}$ Further experiments, including variation of epoch length or event related fMRI studies are needed to clarify the precise phase and time course of activations and their relation to the stimulus.

One potential artifactual source of increased fMRI signal during the resting phase is from involuntary eye movement, which could not be monitored with the apparatus used in this study. However, the extraoccipital responses that we have demonstrated in patients do not include the frontal eye fields or other classic oculomotor control areas, and would not be expected to be activated by eye movements. Furthermore, we think that eye movements are unlikely to have contributed to the difference in activation patterns between patients and normal subjects as patients fixated normally during visual field perimetry, and had no clinical evidence of any eye movement disorder.

There have to date been few other studies of demyelinating optic neuritis using fMRI, and no studies have investigated a directly comparable group of patients after isolated unilateral optic neuritis. Rombouts et $a l^{29}$ studied nine patients with a variable degree of visual recovery from optic neuritis, eight of whom had clinically definite multiple sclerosis, and did not report extraoccipital activation. This could be due to several methodological differences compared with the present study. Firstly, the patients in our study were more homogeneous in their clinical history and MRI appearances, with a uniformly good degree of visual recovery. It is possible that this selected group have different visual activation patterns compared with a heterogeneous group of patients, some of whom had a poor recovery or disseminated disease. Secondly, the stimulus used in the present study was of 20 second duration compared with 40 seconds; it is known that epoch length affects the modulation of the fMRI signal, ${ }^{30}$ and this could have different effects in different parts of the brain. Thirdly, the analysis method we have used takes account of locally variable haemodynamic delay and response shape and allows an accurate estimation of the phase of periodic response in different brain regions; the time shifted boxcar approach used by Rombouts et $a l^{29}$ may not be sensitive to extraoccipital activation if it is phase shifted with respect to the visual stimulus.

Rombouts et $a l^{29}$ reported a reduced volume of visual cortex activation in patients on stimulating both the affected or the unaffected eye in comparison with control subjects. The results of ANOVA comparisons between the generic maps of patients and controls (tables 3 and 4) show a relatively greater response in visual cortical areas in controls compared with patients for both affected and unaffected eyes, in agreement with these findings.

Two other studies of patients with optic neuritis using $\mathrm{fMRI}^{31}{ }^{32}$ studied clinically heterogeneous populations of patients using data acquisition and analysis methods that were not optimised to detect extraoccipital activation. One study included four patients with multiple sclerosis, three of whom had experienced an episode of unilateral optic neuritis ${ }^{31}$ using a surface MR receiver coil which cannot detect activation in anterior brain regions. Another study investigated eight patients (five with definite or probable MS) with varying degrees of clinical recovery after optic neuritis. ${ }^{32}$ In this study images were acquired in the coronal plane with limited brain coverage in the anteroposterior direction; these data do not include anterior brain regions in which we have noted activation in recovered patients.

In summary the present study has disclosed a previously unsuspected degree of functional reorganisation of the brain after acute unilateral optic neuritis implicating a distributed cortical and subcortical network of multimodal areas that are not normally involved in a simple visual stimulation experiment. These areas have extensive visual connections. Whether and how these changes might contribute to the recovery process and its maintenance remains to be determined. Components of this network have been identified in other functional imaging studies as potential areas of cross modal integration between different sensory inputsfor example, when seen speech influences the perception of heard speech. ${ }^{33}$ Our results may 
thus reflect a greater than normal contribution of such higher processing areas (so called top down processing) in patients compared with controls. One possibility is that multimodal regions contribute to suppression of the abnormal input from the affected eye; there is experimental evidence that when the two eyes receive dissimilar visual inputs there is a long latency suppression of response from one eye that may be mediated by cortico-thalamic pathways. ${ }^{34}$ Alternatively, a recent study has reported activation in a network including posterior parietal, occipitotemporal cortex, and anterior insula during a spatial attention task, ${ }^{35}$ and it is possible that activation of these areas in our data reflects increased attention and thus perhaps perceptual enhancement of a pathologically degraded stimulus. The data we have presented cannot rule out a possible contribution from increasing fatigue (or reduced arousal) in patients at the end of the baseline period to the extraoccipital activation, however. At this stage it is clear that temporary visual loss in optic neuritis has previously unexpected long term consequences for the pattern of activation of the brain by simple visual stimuli. An important next step will be to determine the time course of the changes, and their relation to clinical and electrophysiological measures in serial studies from symptom onset.

The NMR research unit is funded by a generous grant from the Multiple Sclerosis Society of Great Britain and Northern Ireland. DJW is supported by the Brain Research Trust. ETB is funded by the Wellcome Trust. We thank Dr PM Matthews, D SJ Jones, Dr AP Leff, and Dr GJM Parker for helpful discussions.

1 McDonald WI. The effects of experimental demyelination on conduction in peripheral nerve: a histological and electrophysiological study. Brain 1963;86:501-24

2 Halliday AM, McDonald WI, Mushin J. Delayed visua evoked response in optic neuritis. Lancet 1972;i:982-5.

3 Jones SJ. Visual evoked potentials after optic neuritis. Effect of time interval, age and disease dissemination. $\mathcal{F}$ Neurol 1993;240:489-94.

4 McLeod JG. Electrophysiological studies in the GuillainBarre syndrome. Ann Neurol 1981:9.S20-7.

5 Werring DJ, Miller DH, Barker GJ, et al. Extensive brain activation following recovery from optic neuritis: a pilot study using functional magnetic resonance imaging. Proc Int Soc Magn Reson Med 1999;2:942.

6 Compston DA, Batchelor JR, Earl CJ, et al. Factors influencing the risk of multiple sclerosis developing in influencing the risk of multiple sclerosis developin

7 Brusa A, Mortimer C, Jones SJ. Clinical evaluation of VEPs to interleaved checkerboard reversal stimulation of central, hemi- and peripheral fields. Electroencephalogr Clin Neurophysiol 1995;96:485-94.

8 Gass A, Barker GJ, MacManus D, et al. High resolution magnetic resonance of the anterior visual pathways in patients with optic neuropathies using fast spin echo and phased array local coils. I Neurol Neurosurg Psychiatry 1995;58:562-5.

9 Bullmore ET, Brammer MJ, Rabe-Hesketh S, et al. Methods for diagnosis and treatment of stimulus-correlated motion in generic brain activation studies using fMRI Human Brain Mapping 1999; 7:38-48.
10 Bullmore ET, Brammer MJ, Williams SCR, et al. Statistical methods of estimation and inference for functional MR image analysis. Magn Reson Med 1996;35:261-77.

11 Bullmore ET, Rabe-Hesketh S, Morris RG, et al. Functional magnetic resonance image analysis of a large-scale neurocognitive network. Neuroimage 1996;4:16-33.

12 Talairach J, Tournoux P. Co-planar stereotaxic atlas of the human brain. New York: Thieme Medical, 1988.

13 Brammer MJ, Bullmore ET, Simmons A, et al. Generic brain activation mapping in functional magnetic resonance imaging: a nonparametric approach. Magn Reson Imaging 1997;15:763-70.

14 Edgington ES. Randomisation tests. New York: Marcel Dekker, 1980.

15 Bullmore ET, Suckling J, Overmeyer S, et al. Global, voxel and cluster tests, by theory and permutation, for a difference between two groups of structural MR images of the brain. IEEE Transactions on Medical Imaging 1999;18: $32-42$.

16 Mesulam M. From sensation to cognition. Brain 1998;121: 1013-52

17 Sherk H. In: Jones EG, Peters A, eds. The claustrum and the cerebral cortex. New York: Plenum Press, 1986.

18 Olsen CR, Graybiel AM. Sensory maps in the claustrum of the cat. Nature 1980;288:479-81.

19 Tsumoto T, Suda K. Effects of stimulation of the dorsocaudal claustrum on activities of striate cortex neurones in the cat. Brain Res 1982;240:345-59.

20 Hadjikhani N, Roland P. Cross-modal transfer of information between the tactile and the visual representations in the human brain: a positron emission tomographic study. $\mathcal{F}$ Neurosci 1998;18:1072-84.

21 Mesulam MM, Mufson EJ. In: Jones EG, Peters A, eds. The insula of Reil in man and monkey: architectonics, connectivity and function. New York: Plenum Press, 1986.

22 Berthier M, Starskein S, Leigurda R. Behavioural effects of damage to the right insula and surrounding regions. Cortex 1987;23:673-8.

23 Mufson EJ, Mesulam MM. Thalamic connections of the insula in the rhesus monkey and comments on the paralimbic connectivity of the medial pulvinar nucleus. F Comp bic connectivity of the med

24 Nieuollon A, Cheramy A, Glowinski J. Release of dopamine evoked by electrical stimulation of the motor and visual areas of the cerebral cortex in both caudate nuclei and in the substantia nigra in the cat. Brain Res 1978;145:69-83.

25 Youl BD, Turano G, Miller DH, et al. The pathophysiology of acute optic neuritis. Brain 1991;114:2437-50.

26 Ciaramitaro VM, Todd WE, Rosenquist AC. Disinhibition of the superior colliculus restores orienting to visual stimuli in the hemianopic field of the cat. F Comp Neurol 1997;387: 568-87.

27 Bandettini P, Wong EC, Binder JR. et al. Functional MR imaging using the BOLD approach. Dynamic characteristics and data analysis. In: Le Bihan D, ed. Diffusion and perfusion magnetic resonance imaging. New York: Raven Press, 1995

28 Bandettini P, EC Wong. In: Schmitt MK, Stehling R, Turner $\mathrm{R}$, eds. Echo-planar imaging: theory, technique and application. Berlin: Springer, 1998

29 Rombouts SARB, Lazeron RHC, Scheltens P, et al. Visual activation patterns in patients with optic neuritis: an fMRI study. Neurology 1998;50:1896-9.

30 Thomas CG, Menon RS. Amplitude response and stimulus presentation frequency response of human primary visual cortex using BOLD EPI at 4T. Magn Reson Med 1998;40: 203-9.

31 Gareau PJ, Gati JS, Menon RS, et al. Reduced visual evoked responses in multiple sclerosis patients with optic neuritis: comparison of functional magnetic resonance imaging and visual evoked potentials. Multiple Scerosis 1999;5:161-4.

32 Langkilde AR, Rostrup E, Frederiksen JL, et al. Functional MR imaging of the visual cortex in patients with previous optic neuritis [abstract]. Proceedings of the International Society of Magnetic Resonance Medicine 1999;2:818.

33 Calvert GA, Brammer MJ, Iversen SD. Crossmodal identification. Trends in Cognitive Sciences 1998;2:247-53.

34 Varela FJ, Singer W. Neuronal dynamics in the visual corticothalamic pathway revealed through binocular rivalry. Exp Brain Res 1987;66:10-20

35 Gitelman DR, Nobre AC, Parrish TB. A large-scale distributed network for covert spatial attention. Brain 1999;122 1093-106. 